\title{
SYMMETRIC FINITE ELEMENT AND BOUNDARY INTEGRAL COUPLING METHODS FOR FLUID-SOLID INTERACTION
}

\author{
BY \\ J. BIELAK AND R. C. MACCAMY \\ Carnegie Mellon University, Pittsburgh, Pennsylvania
}

\begin{abstract}
This paper presents a coupled finite element and boundary integral method for solving the time-periodic oscillation and scattering problem of an inhomogeneous elastic body immersed in a compressible, inviscid, homogeneous fluid. By using integral representations for the solution in the infinite exterior region occupied by the fluid, the problem is reduced to one defined only over the finite region occupied by the solid, with associated nonlocal boundary conditions. This problem is then given a family of variational formulations, including a symmetric one, which are used to derive finite-dimensional Galerkin approximations. The validity of the method is established explicitly, and results of an error analysis are discussed, showing optimal convergence to a classical solution.
\end{abstract}

1. Introduction. We consider the problem of an elastic body immersed in a compressible, inviscid homogeneous fluid. More precisely, we study small time-periodic oscillations and scattering about a constant equilibrium state due to an incident acoustic wave propagating through the fluid. The body may be spatially inhomogeneous. A precise statement appears in Sec. 2. Various physical applications are described, e.g., in $[10,12]$.

The solid-fluid interaction is a special case of a general situation. Let $\Omega$ be a bounded region in space, with boundary $\Gamma$, and let $\Omega^{+}$be the complement of $\Omega$. Suppose one has equations $L u=0$ in $\Omega$ and $L_{+} u=0$ in $\Omega^{+}$with transition conditions across $\Gamma$. The coupling idea is the following. If $L_{+}$is spatially homogeneous then one can apply integral equation methods to $\Omega^{+}$so as to obtain relations between the exterior Cauchy data on $\Gamma$. One then uses the transition conditions to obtain relations on the inner Cauchy data. The result is a problem for $L u=0$ in $\Omega$ with nonlocal boundary conditions which one can then solve by finite element methods. This procedure is often denoted as the coupled boundary element and finite element method for interface problems.

The coupling idea has been used in a great variety of circumstances, starting with [14] in the engineering literature, with [9] apparently providing the first theoretical

Received February 19, 1990.

Supported by the National Science Foundation under Grant DMS 8601288.

(C) 1991 Brown University 
justification. Formal coupling procedures are reviewed, e.g., in [3]. Reference [8] describes and analyzes one coupling method for the elastic solid-fluid problem. Here we present a different one. In fact, in Sec. 3 we present a family of nonlocal problems $\left(\mathrm{P}_{\alpha}\right)$ depending on a parameter $\alpha, 0 \leq \alpha \leq 1$. For each we give a variational formulation $\left(\mathrm{VP}_{\alpha}\right)$. We also give a family of finite-dimensional approximation problems $\left(\mathrm{AVP}_{\alpha \alpha}^{h}\right)$. The choice of $\alpha$ is dictated by what kind of information is of most interest, but when $\alpha=\frac{1}{2}$ the problems $\left(\mathrm{AVP}_{\alpha}^{h}\right)$ are symmetric which will facilitate numerical computation. Our methods were inspired by analogous ones given in [4] for elastic-elastic scattering.

REMARK 1.1. We feel our method has two advantages over that in [8]. It avoids the use of hypersingular integrals and it leads to symmetric finite element equations.

REMARK 1.2. An important feature of the method here and the one in [4] is the symmetry of the resulting Galerkin equations. We are presently performing numerical work on the elastic-elastic problem with good accuracy.

REMARK 1.3. In Sec. 4 and the Appendix we give a proof of the validity of our method, and show the optimum convergence of the Galerkin approximations.

REMARK 1.4. Extensions are possible. If the obstacle is a shell the procedure is essentially the same. In principle one can hope to extend to nonlinear elastic bodies but then the time-periodic theory, in general, no longer applies and one needs a variation of the treatment of the exterior problem with artificial boundaries $[1,2,6]$.

2. Statement of the problem. We suppose $\Omega$ represents a linear, isotropic elastic solid. If $\mathbf{u}$ denotes the displacement and $\Sigma[\mathbf{u}]$ the stress then

$$
\Sigma[\mathbf{u}]=\mu\left(\nabla \mathbf{u}+(\nabla \mathbf{u})^{T}\right)+\lambda \operatorname{div} \mathbf{u} I
$$

where $\mu$ and $\lambda$ can be functions of position $x$. If $\rho_{E}$ is the density, the equation of motion (with no body force) is,

$$
\rho_{E} \mathbf{u}_{t t}=\operatorname{div} \Sigma[\mathbf{u}]
$$

We assume the exterior region $\Omega^{+}$to be filled with a compressible, inviscid fluid. We assume that the fluid remains close to an equilibrium state with constant density and zero velocity. Thus the density $r=\rho_{0}+\rho, \rho \ll 1$ and one has an approximate equation of state $P=f\left(\rho_{0}+\rho\right) \approx f\left(\rho_{0}\right)+f^{\prime}\left(\rho_{0}\right) \rho=P_{0}+c^{2} \rho$ for the pressure, where $c$ is the speed of sound in the linearized fluid. If $\mathbf{v}$ is the velocity $(\mathbf{v} \ll 1)$ then the linearized equations of motion are

$$
\rho_{0} \mathbf{v}_{t}=-\operatorname{grad} P, \quad \rho_{t}+\rho_{0} \operatorname{div} \mathbf{v}=0, \quad \rho=\frac{1}{c^{2}} p .
$$

In terms of pressure alone one has

$$
\frac{1}{c^{2}} p_{t t}=\Delta p, \quad \mathbf{v}_{t}=-\frac{1}{\rho_{0}} \operatorname{grad} p .
$$

The solid-fluid interaction problem involves solving (2.2) in $\Omega$ and (2.4) in $\Omega^{+}$. One needs transition conditions on $\Gamma$. One condition is the continuity of traction. Since the stress in $\Omega^{+}$is simply $-p I$ this yields

$$
\left(\Sigma\left[\mathbf{u}^{-}\right](\mathbf{n})\right) \cdot \mathbf{n}=-p^{+}, \quad\left(\Sigma\left[\mathbf{u}^{-}\right](\mathbf{n})\right) \times \mathbf{n}=\mathbf{0}
$$


where the minus and plus denote interior and exterior limits on $\Gamma$ and $\mathbf{n}$ is the outward unit normal. Since tangential motion of the obstacle will not produce tangential motion of a nonviscous fluid one requires continuity only of the normal component of velocity. This yields the transition condition,

$$
\mathbf{u}_{t t}^{-} \cdot \mathbf{n}=\mathbf{v}_{t}^{+} \cdot \mathbf{n}=-\frac{1}{\rho_{0}} p_{n}^{+} .
$$

We assume that there is an incident fluid motion given by a pressure which has the form $\operatorname{Re}\left(p^{0}(x) e^{i \omega t}\right)$ and we look for the corresponding steady state periodic motion $\mathbf{u}(x, t)=\operatorname{Re}\left(\mathbf{U}(x) e^{i \omega t}\right), p(x, t)=\operatorname{Re}\left(\left(p^{0}(x)+P(x)\right) e^{i \omega t}\right)$ so that $P$ represents the scattered field in $\Omega^{+}$. We impose a radiation condition on $P$. Thus we obtain Problem $\left(\mathrm{P}\left(p^{0}\right)\right)$ :

$$
\begin{gathered}
\operatorname{div} \Sigma[\mathbf{U}]+\rho_{E} \omega^{2} \mathbf{U}=\mathbf{0} \quad \text { in } \Omega ; \quad \Delta P+\frac{\omega^{2}}{c^{2}} P=0 \quad \text { in } \Omega^{+}, \\
\Sigma[\mathbf{U}]^{-}(\mathbf{n}) \cdot \mathbf{n}=-\left(P^{+}+p^{0}\right), \quad\left(\Sigma[\mathbf{U}]^{-}(\mathbf{n})\right) \times \mathbf{n}=\mathbf{0}, \\
\rho_{0} \omega^{2} \mathbf{U}^{-} \cdot \mathbf{n}=\left(P_{n}^{+}+p_{n}^{0}\right) \quad \text { on } \Gamma .
\end{gathered}
$$

$P$ satisfies the radiation condition.

There is a difficulty with uniqueness in $\left(\mathrm{P}\left(p^{0}\right)\right)$.

Theorem 2.1. Suppose $(\mathbf{U}, P)$ is a solution of $P(0)$. Then $P \equiv 0$ and

$$
\operatorname{div} \Sigma[\mathbf{U}]+\rho_{E} \omega^{2} \mathbf{U}=\mathbf{0}, \quad \Sigma[\mathbf{U}]^{-} \cdot \mathbf{n}=0, \quad \mathbf{U}^{-} \cdot \mathbf{n}=0 .
$$

The proof of this theorem is essentially the same as that of Theorem 1.1 of [7]. One uses Green's theorem arguments and Rellich's lemma to conclude that $P \equiv 0$ in $\Omega$.

It is known that for certain regions (2.7) can have nonzero solutions. This appears to be rare but in any event we will rule out this possibility by making the following assumption:

(2.7) has no nontrivial solutions.

3. Nonlocal problems. We start with the exterior region $\Omega^{+}$. Let $\kappa^{2}=\omega^{2} / c^{2}$, the wave number, and put $K(x, y)=-(4 \pi)^{-1}|x-y|^{-1} \exp (i \kappa|x-y|)$. Then we consider the layer potentials,

$$
\mathscr{S}[\phi](x)=\int_{\Gamma} K(x, y) \phi(y) d \sigma_{y} ; \quad \mathscr{D}[\phi](x)=\int_{\Gamma} \frac{\partial}{\partial n_{y}} K(x, y) \phi(y) d \sigma_{y} .
$$

We recall the jump relations

$$
\begin{aligned}
& \mathscr{S}[\phi]^{ \pm}(x)=S[\phi](x), \\
& \mathscr{D}[\phi]^{ \pm}(x)=\mp \frac{1}{2} \phi(x)+D[\phi](x), \\
& \frac{\partial}{\partial n_{x}} \mathscr{S}[\phi]^{ \pm}(x)= \pm \frac{1}{2} \phi+N[\phi](x) \text { on } \Gamma .
\end{aligned}
$$

Here $S, D$, and $N$ are integral operators on $\Gamma$. We summarize well known results for the case of smooth surfaces $\Gamma$. 
LEMMA 3.1. (i) The following are bounded linear mappings $S: H_{r}(\Gamma) \rightarrow H_{r+1}(\Gamma)$; $D: H_{r}(\Gamma) \rightarrow H_{r+2}(\Gamma) ; \quad N: H_{r}(\Gamma) \rightarrow H_{r+2}(\Gamma)$.

(ii) As mappings on $L_{2}(\Gamma), S$ is self adjoint and $D$ and $N$ are adjoint.

(iii) If $\Delta p+\kappa^{2} p=0$ in $\Omega^{+}$and $p$ satisfies a radiation condition, then

$$
p=\mathscr{S}\left[p_{n}^{+}\right]-\mathscr{D}\left[p^{+}\right] .
$$

We also need the following result

Lemma 3.2. Let $\left\{\mu_{n}\right\}$ be the set of eigenvalues of the Laplacian in $\Omega$ with Dirichlet boundary conditions. Then

(i) The mappings $\frac{1}{2} I+D$ and $\frac{1}{2} I+N$ are singular if and only if $\kappa^{2}=-\mu_{n}$ for some $n$.

(ii) If $\frac{1}{2} \psi+N[\psi]=0$ then $\mathscr{S}[\psi]$ is identically zero in $\Omega^{+}$.

Proof. Suppose $\frac{1}{2} \psi+N[\psi]=0$ and put $u=\mathscr{S}[\psi]$. Then $\Delta u+\kappa^{2} u=0$ in $\Omega^{+}$, $u$ satisfies a radiation condition and $u_{n}^{+}=0$ on $\Gamma$. It follows that $u \equiv 0$ in $\Omega^{+}$. Thus $u^{+} \equiv 0$ and, by (3.2), $u^{-} \equiv 0$. Thus we have $\Delta u=-\kappa^{2} u$ in $\Omega, u=0$ on $\Gamma$. It follows that $-\kappa^{2}=\mu_{n}$ for some $n$. Since $N$ and $D$ are adjoint the result follows.

The occurrence of special parameter values is typical of coupling methods and our methods fail for these values. Thus we make an assumption ruling out these cases:

$$
\kappa^{2} \neq-\mu_{n} \text { for any } n \text {. }
$$

(Special techniques for dealing with the case $\kappa^{2}=-\mu_{n}$ have been presented, e.g., in $[5,13]$.) We are now ready to describe our nonlocal problems.

Problem $\left(\mathrm{P}_{0}\left(p^{0}\right)\right)$. Suppose $(\mathbf{U}, P)$ is a solution of $\left(\mathrm{P}\left(p^{0}\right)\right)$. Then by $(3.3)$ and (3.2)

$$
\begin{aligned}
& P=S\left[P_{n}^{+}\right]-\mathscr{D}\left[P^{+}\right] \quad \text { in } \Omega^{+}, \\
& \frac{1}{2} P^{+}=S\left[P_{n}^{+}\right]-D\left[P^{+}\right] \quad \text { on } \Gamma .
\end{aligned}
$$

We put $\lambda=\Sigma\left[\mathbf{U}^{-}\right](\mathbf{n}) \cdot \mathbf{n}$ and insert the transition conditions in (3.4). This yields the problem,

$$
\begin{gathered}
\operatorname{div} \Sigma[\mathbf{U}]+\rho_{E} \omega^{2} \mathbf{U}=\mathbf{0} \quad \text { in } \Omega, \\
\Sigma\left[\mathbf{U}^{-}\right](\mathbf{n}) \cdot \mathbf{n}=\lambda, \quad \Sigma\left[\mathbf{U}^{-}\right](\mathbf{n}) \times \mathbf{n}=\mathbf{0}, \\
\rho_{0} \omega^{2} S\left[\mathbf{U}^{-} \cdot \mathbf{n}\right]+\frac{1}{2} \lambda+D[\lambda]=f=\frac{1}{2} p^{0}+S\left[p_{n}^{0}\right]-D\left[p^{0}\right] \quad \text { on } \Gamma .
\end{gathered}
$$

We have also,

$$
P=\mathscr{S}\left[\rho_{0} \omega^{2} \mathbf{U}^{-} \cdot \mathbf{n}-p_{n}^{0}\right]+\mathscr{D}\left[\lambda+p^{0}\right] \quad \text { in } \Omega^{+} .
$$

REMARK 3.1. If $p^{0}$ satisfies $\Delta p^{0}+\kappa^{2} p^{0}=0$ in $\Omega$ then a Green's theorem argument shows that $f=-p^{0}$.

Problem $\left(\mathrm{P}_{1}\left(p^{0}\right)\right)$. Again suppose $(\mathbf{U}, P)$ is a solution. We assume

$$
P=\mathscr{S}[\psi] \text { in } \Omega^{+} .
$$


Then (3.2) yields

$$
P^{+}=S[\psi], \quad P_{n}^{+}=\frac{1}{2} \psi+N[\psi] \quad \text { on } \Gamma .
$$

Once gain we insert the transition conditions and obtain

$$
\begin{gathered}
\operatorname{div} \Sigma[\mathbf{U}]+\rho_{E} \omega^{2} \mathbf{U}=\mathbf{0} \quad \text { in } \Omega, \\
\Sigma\left[\mathbf{U}^{-}\right](\mathbf{n}) \Sigma\left[\mathbf{U}^{-}\right](\mathbf{n}) \cdot \mathbf{n}=-S[\psi]-p^{0}, \quad \Sigma\left[\mathbf{U}^{-}\right](\mathbf{n}) \times \mathbf{n}=0 \\
-\rho_{0} \omega^{2} \mathbf{U}^{-} \cdot \mathbf{n}+\frac{1}{2} \psi+N[\psi]=-p_{n}^{0} \quad \text { on } \Gamma .
\end{gathered}
$$

Theorem 3.1. Suppose Assumption $\left(\mathrm{A}_{2}\right)$ holds. Then

(i) If $(\mathbf{U}, \lambda)$ is a solution of $\left(\mathrm{P}_{0}\left(p^{0}\right)\right)$ and $P$ is defined by $(3.5)$ then $(\mathbf{U}, P)$ is a solution $\left(\mathrm{P}\left(p^{0}\right)\right)$.

(ii) If $(\mathbf{U}, \psi)$ is a solution $\left(\mathrm{P}_{1}\left(p^{0}\right)\right)$ and $P$ is defined by $(3.6)$ then $(\mathbf{U}, P)$ is a solution of $\left(\mathrm{P}\left(p^{0}\right)\right)$.

Proof. For $\left(\mathrm{P}_{0}\left(p^{0}\right)\right),(3.5)$ and $(3.2)$ yield

$$
P^{+}=\rho_{0} \omega^{2} S\left[\mathbf{U}^{-} \cdot \mathbf{n}\right]-\frac{1}{2} \lambda+D[\lambda]-S\left[p_{n}^{0}\right]-\frac{1}{2} p^{0}+D\left[p^{0}\right]
$$

and the second boundary condition in $\left(\mathrm{P}^{0}\left(p^{0}\right)\right)$ shows that $P^{+}=-\lambda-p^{0}$. Thus (3.5) becomes

$$
P=\mathscr{S}\left[\rho_{0} \omega^{2} \mathbf{U}^{-} \cdot \mathbf{n}-p_{n}^{0}\right]-\mathscr{D}\left[P^{+}\right] .
$$

But $P$ is a solution of $\Delta P+\kappa^{2} P=0$ in $\Omega^{+}$and satisfies a radiation condition. Thus we have also

$$
P=\mathscr{S}\left[P_{n}^{+}\right]-\mathscr{D}\left[P^{+}\right] .
$$

From (3.7) and (3.8) we see that $\chi=\rho_{0} \omega^{2} \mathbf{U} \cdot \mathbf{n}-p_{n}^{0}-P_{n}^{+}$satisfies $\frac{1}{2} \chi+N[\chi]=0$. It follows from $\left(\mathrm{A}_{2}\right)$ that $\chi \equiv 0$.

For $\left(\mathrm{P}_{1}\left(p^{0}\right)\right)$ we have from $(3.6)$

$$
P^{+}=S[\psi], \quad P_{n}^{+}=\frac{1}{2} \psi+N[\psi] .
$$

The boundary conditions for $\left(\mathrm{P}_{1}\left(p^{0}\right)\right)$ then yield $\Sigma[\mathbf{U}](\mathbf{n}) \cdot \mathbf{n}=-P^{+}-p^{0}$ and $\rho_{0} \omega^{2} \mathbf{U}^{-} \cdot \mathbf{n}=P_{n}^{+}+p_{n}^{0}$.

Corollary 3.1. If $\left(A_{1}\right)$ and $\left(A_{2}\right)$ hold $\left(P_{0}(0)\right)$ and $\left(P_{1}(0)\right)$ have only the trivial solutions.

Proof. Suppose $(\mathbf{U}, \lambda)$ solves $\left(\mathrm{P}_{0}(0)\right)$. Then, by Theorem 3.1 , if we define $P$ by (3.5), $\mathbf{U}, P$ is a solution of $(\mathrm{P}(0))$. Hence by Theorem $2.1 \mathrm{U} \equiv \mathbf{0}$ and $P \equiv 0$. $P=\mathscr{S}[-\lambda]$ and $P \equiv 0$ in $\Omega^{+}$implies $P_{n}^{+}=\frac{1}{2} \lambda+N[\lambda]=0$. Hence, by $\left(\mathrm{A}_{2}\right)$, $\lambda \equiv 0$. The proof for $\left(P_{1}(0)\right)$ is the same. 
We now give variational formulations for $\left(\mathrm{P}_{0}\left(p^{0}\right)\right)$ and $\left(\mathrm{P}_{1}\left(p^{0}\right)\right)$, both involving only natural boundary conditions. We take $H$ to be the Hilbert space $H=H_{1}(\Omega) \times$ $L_{2}(\Gamma)$. We introduce the bilinear forms

$$
\begin{aligned}
& A_{11}^{0}(\mathbf{U}, \mathbf{V})=A_{11}^{1}(\mathbf{U}, \mathbf{V})=\int_{\Omega} \Sigma[\mathbf{U}]: \nabla \overline{\mathbf{V}} d x-\omega^{2} \int_{\Omega} \rho_{E} \mathbf{U} \cdot \bar{V} d x, \\
& A_{12}^{0}(\lambda, \mathbf{V})=-\int_{\Gamma} \lambda \overline{\mathbf{V}} \cdot \mathbf{n} d \sigma, \quad A_{12}^{1}(\psi, \mathbf{V})=\int_{\Gamma} S[\psi] \overline{\mathbf{V}} \cdot \mathbf{n} d \sigma, \\
& A_{21}^{0}(\phi, \mathbf{U})=\int_{\Gamma} S\left[\mathbf{n} \cdot \mathbf{U}^{-}\right] \bar{\phi} d \sigma, \quad A_{21}^{1}(\chi, \mathbf{U})=-\int_{\Gamma} \mathbf{U}^{-} \cdot \mathbf{n} \bar{\chi} d \sigma, \\
& A_{22}^{0}(\lambda, \phi)=\frac{1}{\rho_{0} \omega^{2}} \int_{\Gamma}\left(\frac{1}{2} \lambda+D[\lambda]\right) \bar{\phi} d \sigma \\
& A_{22}^{1}(\psi, \chi)=\frac{1}{\rho_{0} \omega^{2}} \int_{\Gamma}\left(\frac{1}{2} \psi+N[\psi]\right) \bar{\chi} d \sigma .
\end{aligned}
$$

We write $\mathscr{U}^{0}=(\mathbf{U}, \lambda), \mathscr{U}^{1}=(\mathbf{U}, \psi), \mathscr{V}^{0}=(\mathbf{V}, \phi), \mathscr{V}^{1}=(\mathbf{V}, \chi)$ and

$$
\begin{aligned}
& \mathscr{A}^{0}\left(\mathscr{U}^{0}, \mathscr{V}^{0}\right)=A_{11}^{0}(\mathbf{U}, \mathbf{V})+A_{12}^{0}(\psi, \mathbf{V})+A_{21}^{0}(\phi, \mathbf{U})+A_{22}^{0}(\lambda, \phi), \\
& \mathscr{A}^{1}\left(\mathscr{U}^{1}, \mathscr{V}^{1}\right)=A_{11}^{1}(\mathbf{U}, \mathbf{V})+A_{12}^{1}(\psi, V)+A_{21}^{1}(\chi, \mathbf{U})+A_{22}^{1}(\psi, \chi) .
\end{aligned}
$$

Finally, we define functionals $F_{0}$ and $F_{1}$ on $H$ by

$$
\begin{gathered}
\left\langle\mathscr{V}^{0}, F_{0}\right\rangle=\frac{1}{\rho_{0} \omega^{2}} \int_{\Gamma} f \bar{\phi} d \sigma \\
\left\langle\mathscr{V}^{1}, F_{1}\right\rangle=-\int_{\Gamma} p^{0} \overline{\mathbf{V}} \cdot \mathbf{n} d \sigma-\frac{1}{\rho_{0} \omega^{2}} \int_{\Gamma} p_{n}^{0} \bar{\chi} d \sigma .
\end{gathered}
$$

Our variational problems are

Find $\mathscr{U}^{0} \in H$ such that $\mathscr{A}^{0}\left(\mathscr{U}^{0}, \mathscr{V}^{0}\right)=\left\langle\mathscr{V}^{0}, F^{0}\right\rangle$ for any $\mathscr{V}^{0} \in H$.

Find $\mathscr{U}^{1} \in H$ such that $\mathscr{A}^{1}\left(\mathscr{U}^{1}, \mathscr{V}^{1}\right)=\left\langle\mathscr{V}^{1}, F_{1}\right\rangle$ for any $\mathscr{V}^{1} \in H$.

The following result is easily verified.

THeOREM 3.2. (i) If $(\mathbf{U}, \lambda)$ solves $\left(\mathrm{P}_{0}\left(p^{0}\right)\right)$ then $\mathscr{U}^{0}=(\mathbf{U}, \lambda)$ solves $\left(\mathrm{VP}_{0}\right)$. If $\mathscr{U}^{0}=(\mathbf{U}, \lambda)$ solves $\left(\mathrm{VP}_{0}\right)$, and $U$ and $\lambda$ are smooth, $(\mathbf{U}, \lambda)$ solves $\left(\mathbf{P}_{0}\left(p^{0}\right)\right)$.

(ii) If $(\mathbf{U}, \psi)$ solves $\left(\mathrm{P}_{1}\left(p^{0}\right)\right)$ then $\mathscr{U}^{1}=(\mathbf{U}, \psi)$ solves $\left(\mathrm{VP}_{1}\right)$. If $\mathscr{U}^{1}=(\mathbf{U}, \psi)$ solves $\left(\mathbf{V P}_{1}\right)$ and $\mathbf{U}$ and $\psi$ are smooth, $(\mathbf{U}, \psi)$ solves $\left(\mathbf{P}_{1}\left(p^{0}\right)\right)$.

4. Numerical discretization. We consider here the numerical implementation of the variational problems described in the previous section.

Suppose that $\Omega^{h}(\Omega)$ and $\Gamma^{h}(\Gamma)$ are finite-dimensional subspaces of $H_{1}(\Omega)$ and $L_{2}(\Gamma)$, depending on a parameter $h$. We set $H^{h}=\Omega^{h}(\Omega) \times \Gamma^{h}(\Gamma)$ and consider the approximate problems

Find $\mathscr{U}_{0}^{h} \in H^{h}$ such that $\mathscr{A}^{0}\left(\mathscr{U}_{0}^{h}, \mathscr{V}_{0}^{h}\right)=\left\langle\mathscr{V}_{0}^{h}, F_{0}\right\rangle$ for any $\mathscr{V}_{0}^{h} \in H^{h}$. 
Find $\mathscr{U}_{1}^{h} \in H^{h}$ such that $\mathscr{A}^{1}\left(\mathscr{U}_{1}^{h}, \mathscr{V}_{1}^{h}\right)=\left\langle\mathscr{V}_{1}^{h}, F_{1}\right\rangle$ for any $\mathscr{V}_{1}^{h} \in H^{h}$.

Suppose $\zeta_{1}, \ldots, \zeta_{N^{h}}$ and $\mu_{1}, \ldots, \mu_{M^{h}}$ are bases for $\Omega^{h}$ and $\Gamma^{h}$ and we write $\mathbf{U}^{h} \in \mathbf{R}^{N^{h}}, \lambda^{h} \in \mathbf{R}^{M^{h}}, \psi^{h} \in \mathbf{R}^{M^{h}}$ for the corresponding expansions. Then the appropriate variational problems are equivalent to algebraic equations of the form,

$$
\begin{aligned}
& \underset{\sim}{\mathscr{B}}{ }_{11}^{0} \mathbf{U}^{h}+\underset{\sim}{\mathscr{B}}{ }_{12}^{0} \lambda^{h}=\mathbf{0} \quad \underset{\sim}{\mathscr{B}}{ }_{11}^{0} N^{h} \times N^{h} \quad \underset{\sim}{\mathscr{B}}{ }_{12}^{0} N^{h} \times M^{h} \\
& \underset{\sim}{\mathscr{B}}{ }_{21}^{0} \mathbf{U}^{h}+\underset{\sim}{\mathscr{P}}{ }_{22}^{0} \lambda^{h}=\mathbf{g}_{2}^{0} \quad \underset{\mathscr{\sim}}{\sim}{ }_{21}^{0} M^{h} \times N^{h} \quad \underset{\mathscr{B}}{\sim}{ }_{22}^{0} M^{h} \times M^{h} \\
& \underset{\sim}{\mathscr{B}}{ }_{11}^{1} \mathbf{U}^{h}+\underset{\sim}{\mathscr{B}}{ }_{12}^{1} \psi^{h}=\mathbf{g}_{1}^{1} \quad \underset{\sim}{\mathscr{B}}{ }_{11}^{1} N^{h} \times N^{h} \quad \underset{\sim}{\mathscr{B}}{ }_{12}^{1} N^{h} \times M^{h} \\
& \underset{\sim}{\mathscr{B}}{ }_{21}^{1} \mathbf{U}^{h}+\underset{\sim}{\mathscr{\sim}}{ }_{22}^{1} \psi^{h}=\mathbf{g}_{2}^{1} \quad \underset{\sim}{\mathscr{B}}{ }_{21}^{1} M^{h} \times N^{h} \quad \underset{\sim}{\mathscr{B}}{ }_{22}^{0} M^{h} \times M^{h}
\end{aligned}
$$

REMARK 4.1. If one is primarily interested in the inner region $\Omega,\left(\mathrm{P}_{0}\left(p^{0}\right)\right)$ is useful since it produces the interior stress and boundary traction as solutions. The exterior field on the other hand requires two integrations (3.5). If the primary interest is in $\Omega^{+},\left(\mathrm{P}_{1}\left(p^{0}\right)\right)$ is better since the exterior field requires only one integration (3.6).

REMARK 4.2. It is interesting to note that one has a whole family of problems. Since $A_{11}^{0}(\mathbf{U}, \mathbf{V})=A_{11}^{1}(\mathbf{U}, \mathbf{V})$ we can take convex combinations of $\left(\mathrm{P}_{0}\left(p^{0}\right)\right)$ and $\left(\mathrm{P}_{1}\left(p^{0}\right)\right)$. Thus one takes $H=H_{1}(\Omega) \times L_{2}(\Gamma) \times L_{2}(\Gamma)$ with $\mathscr{U}=(\mathbf{U}, \lambda, \psi), \mathscr{V}=$ $(\mathbf{V}, \phi, \xi)$ and

$$
\begin{gathered}
\mathscr{A}^{\alpha}(\mathscr{U}, \mathscr{V})=(1-\alpha) \mathscr{A}^{0}((\mathbf{U}, \mathbf{V}),(\lambda, \phi))+\alpha A^{1}((\mathbf{U}, \mathbf{V}),(\psi, \xi)), \\
\left\langle\mathscr{V}, F_{\alpha}\right\rangle=(1-\alpha)\left\langle(\mathbf{V}, \phi), F_{0}\right\rangle+\alpha\left\langle(\mathbf{V}, \chi), F_{1}\right\rangle,
\end{gathered}
$$

to obtain

Find $\mathscr{U} \in H$ such that $\mathscr{A}^{\alpha}(\mathscr{U}, \mathscr{V})=\left\langle\mathscr{V}, F_{\alpha}\right\rangle$ for any $\mathscr{V} \in H$.

We now have two boundary functions to be determined and the structure of the finite-dimensional approximation equations will be

$$
\begin{aligned}
& \underset{\sim}{\mathscr{B}}{ }_{11}^{0} \mathbf{U}^{h}+(1-\alpha) \underset{\sim}{\mathscr{B}}{ }_{12}^{0} \lambda^{h}+\alpha \underset{\sim}{\mathscr{B}}{ }_{12}^{1} \psi^{h}=\alpha \mathbf{g}_{1}^{1}, \\
& \alpha \underset{\sim}{\mathscr{R}}{ }_{21}^{1} \mathbf{U}^{h}+\alpha \underset{\sim}{\mathscr{B}}{ }_{22}^{1} \boldsymbol{\psi}^{h}=\alpha \mathbf{g}_{2}^{1}, \\
& (1-\alpha) \underset{\sim}{\mathscr{B}}{ }_{21}^{0} \mathbf{U}^{h}+(1-\alpha) \underset{\sim}{\mathscr{B}}{ }_{22}^{p} \lambda^{h}=(1-\alpha) \mathbf{g}_{2}^{0} .
\end{aligned}
$$

If we choose $\alpha=\frac{1}{2}$ and use real basis elements then it is quite easy to verify that the system $\left(\mathrm{E}_{\alpha}^{h}\right)$ is symmetric; that is:

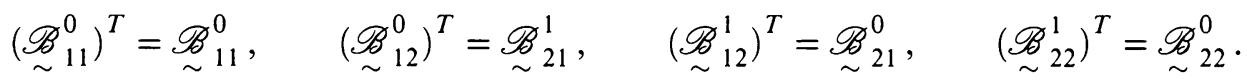

Let us verify one of these. We have

$$
\begin{aligned}
\left(\mathscr{B}_{22}^{1}\right)_{i k}- & =A_{22}^{1}\left(\mu_{k}, \mu_{i}\right)=\frac{1}{\rho_{0} \omega^{2}} \int_{\Gamma}\left(\frac{1}{2} \mu_{k}+N\left[\mu_{k}\right]\right) \mu_{i} d \sigma \\
& =\frac{1}{\rho_{0} \omega^{2}} \int_{\Gamma}\left(\frac{1}{2} \mu_{i}+D\left[\mu_{i}\right]\right) \mu_{k} d \sigma=A_{22}^{0}\left(\mu_{i}, \mu_{k}\right)=\left(\mathscr{B}_{22}^{0}\right)_{k i} .
\end{aligned}
$$


REMARK 4.3. We observe also that our procedures are well adapted to standard software. For instance, we can solve $\left(E_{0}^{h}\right)$ by condensation. The second equation can be solved in the form,

$$
\lambda=\left(\underset{\sim}{\mathscr{B}_{22}^{0}}\right)^{-1} \mathbf{g}_{2}^{0}-\left(\underset{\sim}{\mathscr{B}_{22}^{-1}}\right) \mathscr{B}_{\sim 21}^{0} \mathbf{U}^{h} .
$$

The inverse $\left(\mathscr{B}_{22}^{0}\right)^{-1}$ is a standard one from boundary integral equation methods. One can then insert (4.1) into the first equation to obtain equations of the form

$$
\mathscr{B}_{11}^{0} \mathbf{U}^{h}+\underset{\sim}{\mathscr{C}} \mathbf{U}^{h}=\mathbf{g}_{0} \text {. }
$$

Here $\mathscr{C}=-\mathscr{B}_{12}^{0}\left(\mathscr{B}_{22}^{0}\right)^{-1} \mathscr{B}_{21}^{0}$ and $\mathbf{g}_{0}=\mathscr{B}_{12}^{0}\left(\mathscr{B}_{22}^{0}\right)^{-1} \mathbf{g}_{2}^{0}$, and $\mathscr{B}_{11}^{0}$ is the standard dynamic stiffness, or impedance, matrix for interior elastic problems. This matrix is sparse.

Similarly, by condensing $\psi^{h},\left(E_{1}^{h}\right)$ leads to an equation of the form

$$
\mathscr{B}_{11}^{0} \mathbf{U}^{h}+\mathscr{\mathscr { C }}^{T} \mathbf{U}^{h}=\mathbf{g}_{1}
$$

while $\left(E_{1 / 2}^{h}\right)$ yields the equation

$$
\mathscr{B}_{11}^{0} \mathbf{U}^{h}+\frac{1}{2}\left(\underset{\sim}{\mathscr{C}}+\underset{\sim}{\mathscr{C}^{T}}\right) \mathbf{U}^{h}=\frac{1}{2}\left(\mathbf{g}_{0}+\mathbf{g}_{1}\right) .
$$

It is worth noting that the appearance of the adjoint matrices $\underset{\sim}{\mathscr{C}}$ and $\mathscr{C}^{T}$ in (4.3) and (4.4) is a direct consequence of the adjointness of the linear mappings $D$ and $N$, which occur, respectively, in the formulation of problems $\left(\mathbf{P}_{0}\right)$ and $\left(\mathbf{P}_{1}\right) \cdot\left(\mathbf{P}_{0}\right)$ is based on the integral representation (3.4) for the fluid pressure which introduced $D$, while $\left(\mathrm{P}_{1}\right)$ uses the single-layer representation (3.6) from which $N$ arises. (These two alternatives are denoted as the direct and the indirect formulations in the engineering literature [14].) It is then clear why, whereas each problem $\left(P_{0}\right)$ and $\left(P_{1}\right)$, by itself, leads to a nonsymmetric system of the finite-dimensional approximation equations (4.3), (4.4), their average (4.5) is symmetric. It is this form of the discretized equations which is most convenient for computations. All the elements of the matrices $\underset{\sim}{\mathscr{C}}, \mathscr{C}^{T}$, and $\frac{1}{2}\left(\underset{\sim}{\mathscr{C}}+\mathscr{C}^{T}\right)$ are zero except for those elements associated with the components of $\mathbf{U}^{h}$ located on $\Gamma$. Each of these matrices represents the impedance of the fluid at the interface with the solid, and constitutes, in effect, a discretized nonlocal absorbing boundary. The terms $\mathbf{g}_{0}$ and $\mathbf{g}_{1}$ represent effective forces applied on $\Gamma$, due to the incident wave $p^{0}$. After solving for $\mathbf{U}^{h}, \lambda^{h}$ and $\psi^{h}$ can be evaluated by back substitution, using (4.2) for $\lambda^{h}$ and a similar equation for $\psi^{h}$. The scattered field $P$ can be calculated using (4.2) and $\lambda^{h}$ and a similar equation for $\psi^{h}$. The scattered field $P$ can then be calculated from (3.5) or, more simply, from (3.6).

5. Existence and regularity. Our first goal here is to validate the procedures in the preceding section. The proof of the validity of the coupling methods rests on the following abstract result. Let $H$ be a Hilbert space, $A$ a bounded bilinear form on $H \times H$ and $F$ an element of $H^{\prime}$, the dual of $H$. 
Suppose that $A=A_{0}+A_{1}$ where

There exists $k>0$ such that $A_{0}(u, u) \geq k\|u\|^{2}$ for any $y \in H$.

For any bounded sequence $\left\{u_{k}\right\}$ in $H$ there exists a subsequence

$u_{k_{j}}$ and a $u \in H$ such that $A_{1}\left(u_{k_{j}}, v\right) \rightarrow A_{1}(u, v)$ for any $v \in H$.

Consider the variational problem

Find $u \in H$ such that $A(u, v)=\langle v, F\rangle$ for any $v \in H$.

THEOREM A. If $A(u, v)=0$ for all $v$ implies $u=0$ then (VP) has a unique solution $u$ and $F \rightarrow u$ is a bounded map from $H^{\prime} \rightarrow H$.

Suppose that one has a family $S^{h}$ of finite-dimensional subspaces contained in $H$ and one considers the approximate variational problems:

Find $u^{h} \in S^{h}$ such that $A\left(u^{h}, v^{h}\right)=\left\langle v^{h}, F\right\rangle$ for any $v^{h} \in S^{h}$.

We suppose that the $S^{h}$ approximate $H$ in the following sense:

Given any $\varepsilon>0$ there is an $h_{0}>0$ such that for any $h<h_{0}$ and $u \in H$ there is a $w^{h} \in S^{h}$ such that $\left\|u-w^{h}\right\|<\varepsilon$.

THEOREM B. If $A(u, v)=0$ for all $v$ implies $u=0$ then

(i) There exists an $h_{0}>0$ such that $\left(\mathrm{AVP}_{h}\right)$ has a unique solution $u^{h}$ for any $h<h_{0}$.

(ii) If $u$ is the solution of (VP) $\left\|u-u^{h}\right\| \rightarrow 0$ as $h \downarrow 0$.

Theorem $\mathrm{A}$ is an easy consequence of the Lax-Milgram lemma and Riesz-Schauder theory. Theorem B is fairly well known (see for instance [7] and [11]) but for completeness we sketch the proof in an appendix. The results in the Appendix also show that one has optimal convergence. For instance, if one uses piecewise quadratic approximations in $\Omega$ and piecewise linear approximations on $\Gamma$ one obtains $O\left(h^{2}\right)$ in $H_{1}(\Omega)$ and $O\left(h^{3}\right)$ in $L_{2}(\Gamma)$.

In proving the validity of our procedure, $\left(\mathrm{P}_{0}\left(p^{0}\right)\right)$ and $\left(\mathrm{P}_{1}\left(p^{0}\right)\right)$ can be treated in the same way and both begin with the same observation. We write,

$$
\begin{gathered}
A_{11}^{0}(\mathbf{U}, \mathbf{V})=A_{11}^{1}(\mathbf{U}, \mathbf{V})=A_{0}(\mathbf{U}, \mathbf{V})+A_{1}(\mathbf{U}, \mathbf{V}), \\
A_{0}(\mathbf{U}, \mathbf{V})=\int_{\Omega}(\Sigma[\mathbf{U}]: \nabla \overline{\mathbf{V}}+m \mathbf{U} \cdot \mathbf{V}) d x, \\
A_{1}(\mathbf{U}, \mathbf{V})=-\int_{\Omega} m \mathbf{U} \overline{\mathbf{V}} d x-\omega^{2} \int_{\Omega} \rho_{E} \mathbf{U} \overline{\mathbf{V}} d x .
\end{gathered}
$$

For $m$ sufficiently large, Korn's second inequality implies that there is a $k>0$ such that

$$
A_{0}(\mathbf{U}, \mathbf{U}) \geq k\|\mathbf{U}\|_{H_{1}(\Omega)} .
$$

The form $A_{1}$ satisfies (II). If $\left\{\mathbf{U}_{h}\right\}$ is a bounded sequence in $H_{1}(\Omega)$ then there is a subsequence $U_{k_{j}}$ which converges weakly in $H_{1}(\Omega)$ and strongly in $L_{2}(\Omega)$ to $\mathbf{U} \in H_{1}(\Omega)$; hence $A_{1}\left(\mathbf{U}_{k_{j}}, \mathbf{V}\right) \rightarrow A_{1}(\mathbf{U}, \mathbf{V})$ for any $\mathbf{V} \in H_{1}(\Omega)$. 
Consider first $\left(\mathrm{VP}_{0}\right)$. We decompose $\mathscr{A}^{0}(\mathscr{U}, V)$ in the form

$$
\begin{aligned}
& \mathscr{A}^{0}(\mathscr{U}, \mathscr{V})=\mathscr{A}_{0}^{0}(\mathscr{U}, \mathscr{V})+\mathscr{A}_{1}^{0}(\mathscr{U}, \mathscr{V}), \\
& \mathscr{A}_{0}^{0}(\mathscr{U}, \mathscr{V})=A_{0}(\mathbf{U}, \mathbf{V})+\frac{1}{\rho_{0} \omega^{2}} \int_{\Gamma} \lambda \bar{\phi} d \sigma, \\
& \mathscr{A}_{1}^{0}(\mathscr{U}, \mathscr{V})=A_{1}(\mathbf{U}, \mathbf{V})+A_{12}^{0}(\lambda, \mathbf{V})+A_{21}^{0}(\phi, \mathbf{V})+\frac{1}{\rho_{0} \omega^{2}} \int_{\Gamma} D[\lambda] \bar{\phi} d \sigma .
\end{aligned}
$$

From (4.2) we see that $\mathscr{A}_{0}^{0}$ satisfies (I). We assert that $\mathscr{A}_{1}^{0}$ satisfies (II). We have already seen this for $A_{1}(U, V)$ and we consider the remaining terms under the assumption that $\mathscr{U}_{k}=\left(\mathbf{U}_{k}, \lambda_{k}\right)$ with $\left\{U_{k}\right\}$ a bounded sequence in $H_{1}(\Omega)$ and $\lambda_{k}$ a bounded sequence in $L_{2}(\Gamma)$. Then we have that $\left\{\left.\mathbf{U}_{k}\right|_{\Gamma}\right\}$ is a bounded sequence in $H^{1 / 2}(\Gamma)$. We conclude that there is a subsequence $k_{j}$ such that

$\mathbf{U}_{k_{j}} \quad$ converges weakly in $H_{1}(\Omega)$ to $\mathbf{U}$,

$\left.\mathbf{U}_{k_{j}}\right|_{\Gamma} \quad$ converges weakly in $H_{1 / 2}(\Gamma)$ to $\left.\mathbf{U}\right|_{\Gamma}$,

$\left.\mathbf{U}_{k_{j}}\right|_{\Gamma} \quad$ converges strongly in $L_{2}(\Gamma)$ to $\left.U\right|_{\Gamma}$,

$\lambda_{k_{j}} \quad$ converges weakly to $\lambda$ in $L_{2}(\Gamma)$.

Consider the individual terms

$$
\begin{aligned}
& A_{12}^{0}\left(\lambda_{k_{j}}, \mathbf{V}\right)=-\int_{\Gamma} \lambda_{k_{j}} \bar{V} \cdot \mathbf{n} d \sigma \rightarrow-\int_{\Gamma} \lambda \overline{\mathbf{V}} \cdot \mathbf{n} d \sigma, \\
& A_{21}^{0}\left(\phi, \mathbf{U}_{k_{j}}\right)=\int_{\Gamma} S\left[\mathbf{n} \cdot \mathbf{U}_{k_{j}}\right] \bar{\phi} d \sigma .
\end{aligned}
$$

$S$ is a bounded map from $L_{2}(\Gamma)$ into $H_{1}(\Gamma)$; hence $S\left[\mathbf{n} \cdot \mathbf{U}_{k_{j}}\right]$ converges strongly to $S[\mathbf{n} \cdot \mathbf{U}]$ in $L_{2}(\Gamma)$ and $A_{21}^{0}\left(U, \mathbf{U}_{k_{j}}\right) \rightarrow A_{21}^{0}(\phi, U)^{*}$.

$$
A_{22}^{0}\left(\lambda_{k_{j}}, \phi\right)=\frac{1}{\rho_{0} \omega^{2}} \int_{\Gamma}\left(\frac{1}{2} \lambda_{k_{j}}+D\left[\lambda_{k_{j}}\right]\right) \bar{\phi} d \sigma .
$$

$D$ is a bounded map from $L_{2}(\Gamma)$ into $H_{2}(\Gamma)$ and $\lambda_{k_{j}}$ is bounded in $L_{2}(\Gamma)$ and converges weakly to $\lambda$; hence we can assume (with possibly another subsequence) that $D\left(\lambda_{k_{j}}\right)$ converges strongly to $\lambda$ in $L_{2}(\Gamma)$. Thus $A_{22}^{0}\left(\lambda_{k j}, \phi\right) \rightarrow A_{22}(\lambda, \phi){ }^{1}$

We have thus shown that conditions (I) and (II) are satisfied. If we take $p^{0} \in$ $H_{1}^{\text {loc }}\left(\Omega^{+}\right)$with $\Delta p^{0}+\kappa^{2} p^{0}=0$ then we have $\left.p^{0}\right|_{\Gamma} \in H_{1 / 2}(\Gamma),\left.p_{n}^{0}\right|_{\Gamma} \in H_{-1 / 2}(\Gamma)$ so that $F_{0} \in H_{1}$ and we can use Theorems $\mathrm{A}$ and B. From Corollary 3.1 we see that, under assumptions $\left(\mathrm{A}_{1}\right),\left(\mathrm{A}_{2}\right), \mathscr{A}^{0}(\mathscr{U}, \mathscr{V})=0$ implies $\mathscr{U}=0$; hence, Theorem $A$ implies the existence of a unique solution of $\left(\mathrm{VP}_{0}\right)$. If, in addition, (III) holds Theorem B implies the convergence of Galerkin's method.

The argument for $\left(\mathrm{VP}_{1}\right)$ is the same with the same conclusions.

\footnotetext{
${ }^{1}$ This idea comes from [11].
} 
Under the hypotheses (I) and (II) and assuming $p^{0} \in H_{1}^{\text {loc }}\left(\Omega^{+}\right)$we obtain a generalized solution of $\left(\mathrm{P}_{0}\left(p^{0}\right)\right)$ or $\left(\mathrm{P}_{1}\left(p^{0}\right)\right)$ with $\mathrm{U} \in H_{1}(\Omega)$ and $P \in H_{1}^{\text {loc }}\left(\Omega^{+}\right)$. Because of the special form of these problems it is not difficult to obtain regularity results by bootstrapping. We illustrate with $\left(\mathrm{P}_{0}\left(p^{0}\right)\right)$. Suppose $p^{0} \in H_{r}^{\text {loc }}>\left(\Omega^{+}\right)$with $r>1$. Then $\left.p^{0}\right|_{\Gamma} \in H_{r-1 / 2}(\Gamma)$ and $p_{n}^{0} \in H_{r-3 / 2}(\Gamma)$; hence $f=-\frac{1}{2} p^{0}+\mathscr{S}\left[p_{n}^{0}\right]-D\left[p_{0}\right] \in$ $H_{r-1 / 2}(\Gamma)$. If $\mathbf{U} \in H_{1}(\Gamma)$ we have $\mathbf{U}^{-} \cdot \mathbf{n} \in H_{1 / 2}(\Gamma)$ and $\mathscr{S}\left[\mathbf{U}^{-} \cdot \mathbf{n}\right] \in H_{3 / 2}(\Gamma)$,

$$
\lambda=\left(\frac{1}{2} I+D\right)^{-1}\left\{f-\rho_{0} \omega^{2} S\left[\mathbf{U}^{-} \cdot \mathbf{n}\right]\right\} \in H_{S}(\Gamma) s=\min \left(r-\frac{1}{2}, \frac{3}{2}\right) .
$$

On the other hand we have

$$
\begin{gathered}
\operatorname{div} \Sigma[\mathbf{U}]+\rho \omega^{2} \mathbf{U}=0 \quad \text { in } \Omega, \\
\Sigma[\mathbf{U}](\mathbf{n}) \cdot \mathbf{n}=\lambda, \quad \Sigma[\mathbf{U}](\mathbf{n}) \times \mathbf{n}=\mathbf{0} .
\end{gathered}
$$

Hence $\mathbf{U} \in H_{s+3 / 2}(\Omega)$. Take $r>2$, then we have, at least, $\mathbf{U} \in H_{3}(\Omega)$. If $r$ is arbitrarily large we can continue this process and show that $\mathbf{U} \in H_{k}(\Omega), \lambda \in$ $H_{k-3 / 2}^{\text {loc }}(\Gamma)$ for $k$ arbitrarily large, thus establishing that $(\mathbf{U}, \lambda)$ is a classical solution of $\left(\mathrm{P}_{0}\left(p^{0}\right)\right)$ and, accordingly, that we have a classical solution of $\left(\mathrm{P}\left(p^{0}\right)\right)$.

Appendix. Proof of Theorem B. We begin by defining the Galerkin operator $G_{0}^{h}: H$ $\rightarrow S^{h}$ by $G_{0}^{h} \mathbf{u}=\tilde{u}^{h}$ where

$$
A_{0}\left(\tilde{u}^{h}, v^{h}\right)=A_{0}\left(u, v^{h}\right) \quad \text { for any } v^{h} .
$$

If we expand $\tilde{u}^{h}$ in terms of the basis elements of $S^{h}$, (A.1) becomes a system of linear algebraic equations and condition (I) guarantees that this system has a solution so $G_{0}^{h}$ is well defined. For any $w^{h} \in S^{h}$ we obtain from (A.1),

$$
A_{0}\left(u^{h}-w^{h}, v^{h}\right)=A_{0}\left(u-w^{h}, v^{h}\right),
$$

hence, by (I),

$$
\left\|u^{h}-w^{h}\right\| \leq \frac{1}{h} \bar{a}\left\|u-w^{h}\right\|,
$$

where $\bar{a}$ is an upper bound for $A_{0}$. Thus

$$
\left\|G_{0}^{h} u-u\right\| \leq K\left\|u-w^{h}\right\| \quad \text { for any } u \in H, w^{h} \in S^{h} .
$$

Hence condition (III) implies that $\left\|G_{0}^{h} u-u\right\| \rightarrow 0$ as $h \downarrow 0$ for any $u \in H$, that is, $G_{0}^{h}$ converges strongly to $I$ as $h \downarrow 0$. Moreover we can put $w^{h}=0$ in (A.2) and conclude that $\left\|G_{0}^{h} u-u\right\| \leq K\|u\|$ for any $u \in H$. It follows that $\left\|G_{0}^{h}\right\|$ is uniformly bounded.

Next we consider $A_{1}$. We note that, for each $u \in H$ the map $v \rightarrow A_{1}(u, v)$ is bounded and linear, hence we can define a map $J: H \rightarrow H^{\prime}$ by

$$
\langle v, J u\rangle=A_{1}(u, v) \quad \text { for any } v \in H .
$$

Condition (III) states that $J$ is a compact map. 
Theorem A states that the solution of (VP) defines a bounded solution map $\mathscr{S}$ from $H^{1}$ into $H$ by the equation

$$
A(\mathscr{S} F, v)=\langle v, F\rangle \quad \text { for any } v .
$$

Consider the map $\mathscr{S} J\left(I-G_{0}^{h}\right)$. This is a map from $H$ into itself and we assert that its norm tends to zero as $h \downarrow 0$. Suppose not. Then we find an $\varepsilon>0$ and a sequence $h_{n} \downarrow 0$ with $u_{n} \in H,\left\|u_{n}\right\|=1$ such that $\left\|\mathscr{S} J\left(I-G_{0}^{h}\right) u_{n}\right\| \geq \varepsilon$. Since $\left\|I-G_{0}^{h}\right\|$ is uniformly bounded $\left(I-G_{0}^{h}\right) u_{n}=w_{n}$ is a bounded sequence in $H$ and by the definition (A.1) of $G_{0}^{h}$ one has

$$
A_{0}\left(w_{u}, v^{h}\right)=0 \quad \text { for any } v^{h} \in S^{h} .
$$

Now $\left\{w_{n}\right\}$ is bounded in $H$, hence there is a subsequence $w_{n_{k}}$ which converges weakly in $H$ to $w$. But then (A.5) implies $A_{0}\left(w, v^{h}\right)=0$ for any $v^{h} \in S^{h}$ which, in view of (III), implies $A_{0}(w, v)=0$ for any $v \in S$. Hence by (I) $w=0$. Thus $w_{n}$ converges weakly to zero. But $J$ is compact so $J w_{n}$ converges strongly to zero and $\mathscr{S}$ is bounded so $\mathscr{S} J\left(I-G_{0}^{h}\right) u_{n}$ converges strongly to zero which contradicts $\left\|\mathscr{S} J\left(I-G_{0}^{h}\right) u_{n}\right\| \geq \varepsilon$.

Since $\left\|\mathscr{S} J\left(I-G_{0}^{h}\right)\right\|$ tends to zero as $h \rightarrow 0$ we can find an $h_{0}$ sufficiently small that $\left(I-\mathscr{S} J\left(I-G_{0}^{h}\right)\right)^{-1}$ exists for $h<h_{0}$ and we can define

$$
G^{h}=G_{0}^{h}\left[I-\mathscr{S} J\left(O-G_{0}^{h}\right)\right]^{-1} \text {. }
$$

We assert that

$$
A\left(G^{h} u, v^{h}\right)=A\left(u, v^{h}\right) \quad \text { for any } v^{h} \in S^{h},
$$

that is, $G^{h}$ is the Galerkin operator for $A$. To verify (A.3) put

$$
z=\left(I-\mathscr{S} J\left(I-G_{0}^{h}\right)\right)^{-1} u .
$$

Then we have

$$
z-u=\mathscr{S} J\left(I-G_{0}^{h}\right) z .
$$

By (A.3) and (A.2) this means

$$
\begin{aligned}
A(z, v) & =\left\langle v, J\left(I-G_{0}^{h}\right) z\right\rangle+A(u, v) \\
& =A_{1}\left(\left(I-G_{0}^{h}\right) z, v\right)+A(u, v)
\end{aligned}
$$

or

$$
A_{0}(z, v)+A_{1}\left(G_{0}^{h} z, v\right)=A(u, v) .
$$

We put $v^{h}$ in (A.7). Then $A_{0}\left(z, v^{h}\right)=A_{0}\left(G_{0}^{h} z, v^{h}\right)$ and (A.8) yields

$$
A\left(G_{0}^{h} z, v^{h}\right)=A\left(u, v^{h}\right) \quad \text { for any } v^{h} \in S^{h}
$$

and this is formula (A.5).

(A.5) gives the result we want. For a solution $u$ of (VP) $\left\langle v^{n}, F\right\rangle=A(u, v)$ and (A.5) says that the Galerkin solution is

$$
u^{h}=G_{0}^{h}\left(I-S J\left(I-G_{0}^{h}\right)^{-1} \mathscr{S} F\right) .
$$


The operator in (A.7) tends to $I$ as $h \downarrow 0$ confirming that $u^{h} \rightarrow u$. This completes the proof.

\section{REFERENCES}

[1] A. Barry, J. Bielak, and R. C. MacCamy, On absorbing boundary conditions for wave propagation, J. Comput. Phys 79, 449-468 (1988)

[2] A. Bayliss and E. Turkel, Radiation boundary conditions for wave-like equations, Comm. Pure Appl. Math. 33, 707-725 (1980)

[3] D. E. Beskos, Potential theory, Boundary Element Methods in Mechanics, (D. E. Beskos, ed.), Elsevier, Amsterdam, 1987, pp. 23-106

[4] J. Bielak and R. C. MacCamy, An exterior interface problem in two-dimensional elastodynamics, Quart. Appl. Math. 41, 143-159 (1983)

[5] A. J. Burton and G. F. Miller, The application of integral equation methods to the numerical solutions of some exterior boundary-value problems, Proc. Royal Soc. London Ser. A 323, 201-210 (1971)

[6] B. Engquist and A. Majda, Absorbing boundary conditions for the numerical simulation of waves, Math. Comp. 31, 629-651 (1977)

[7] S. Hildebrandt and E. Weinholz, Construction proofs of representation theorems in separable Hilbert space, Comm. Pure Appl. Math. 17, 369-373 (1964)

[8] G. Hsiao, The coupling of BEM and FEM-A brief review, Boundary Elements X, Vol.1, SpringerVerlag, New York, 1988, pp. 431-445

[9] C. Johnson and J. C. Nedelec, On the coupling of boundary integral and finite element methods, Math. Comp. 35, 1063-1079 (1980)

[10] M. C. Junger and D. Feit, Sound, Structures and Their Interaction, MIT Press, Cambridge, MA, 1986

[11] R. C. MacCamy and E. Stephan, A boundary element method for an exterior problem for threedimensional Maxwell's equation, Appl. Anal 10, 141-163 (1983)

[12] G. S. Sammelmann, D. H. Trivett, and R. H. Hackman, The acoustic scattering by a submerged spherical shell I: The bifurcation of the dispersion curve for the spherical antisymmetric Lamb wave, J. Acoust. Soc. Amer. 85, 114-124 (1989)

[13] H. A. Schenk, Improved integral formulation for acoustic radiation problems, J. Acoust. Soc. Amer. 44, 41-58 (1968)

[14] O. C. Zienkiewicz, D. W. Kelly, and P. Bettes, The coupling of the finite element method and boundary solution procedures, Internat. J. Numer. Methods Engrg. 11, 335-375 (1977) 